\title{
Radio detections towards unidentified variable EGRET sources ${ }^{\star}$
}

\author{
J. M. Paredes ${ }^{1}$, J. Martî ${ }^{2,7}$, C. H. Ishwara-Chandra ${ }^{3}$, D. F. Torres ${ }^{4}$, G. E. Romero ${ }^{5,8, \star \star}$, J. A. Combi ${ }^{2,7}$, \\ V. Bosch-Ramon ${ }^{6}$, A. J. Muñoz-Arjonilla ${ }^{2,7}$, and J. R. Sánchez-Sutil ${ }^{7}$
}

1 Departament d'Astronomia i Meteorologia and Institut de Ciències del Cosmos (ICC), Universitat de Barcelona (UB/IEEC), Martí i Franquès 1, 08028 Barcelona, Spain e-mail: jmparedes@ub.edu

2 Departamento de Física, EPS, Universidad de Jaén, Campus Las Lagunillas s/n, Edif. A3, 23071 Jaén, Spain e-mail: [jmarti; jcombi;ajmunoz]@ujaen.es

3 NCRA, TIFR, Post Bag 3, Ganeshkhind, Pune-411 007, India e-mail: ishwar@ncra.tifr.res.in

4 ICREA \& Institut de Ciencies de l'Espai, Campus UAB, Facultat de Ciencies, Universitat Autònoma de Barcelona Torre C5, parell, 2da planta, Bellaterra, 08193 Barcelona, Spain

e-mail:dtorres@aliga.ieec.uab.es

5 Instituto Argentino de Radioastronomía, C.C.5, (1894) Villa Elisa, Buenos Aires, Argentina e-mail: romero@iar.unlp.edu.ar

6 Max Planck Institut für Kernphysik, Saupfercheckweg 1, Heidelberg 69117, Germany

e-mail: vbosch@mpi-hd.mpg.de

7 Grupo de Investigación FQM-322, Universidad de Jaén, Campus Las Lagunillas s/n, Edif. A3, 23071 Jaén, Spain e-mail: jrssutil@hotmail.com

8 Facultad de Ciencias Astronómicas y Geofísicas, UNLP, Paseo del Bosque, 1900 La Plata, Argentina e-mail: romero@fcaglp.unlp.edu.ar

Received 17 July 2007 / Accepted 7 February 2008

\begin{abstract}
Context. A considerable fraction of the $\gamma$-ray sources discovered with the Energetic Gamma-Ray Experiment Telescope (EGRET) remain unidentified. The EGRET sources that have been properly identified are either pulsars or variable sources at both radio and gamma-ray wavelengths. Most of the variable sources are strong radio blazars. However, some low galactic-latitude EGRET sources, with highly variable $\gamma$-ray emission, lack any evident counterpart according to the radio data available until now.

Aims. The primary goal of this paper is to identify and characterise the potential radio counterparts of four highly variable $\gamma$-ray sources in the galactic plane through mapping the radio surroundings of the EGRET confidence contours and determining the variable radio sources in the field whenever possible.

Methods. We have carried out a radio exploration of the fields of the selected EGRET sources using the Giant Metrewave Radio Telescope (GMRT) interferometer at $21 \mathrm{~cm}$ wavelength, with pointings being separated by months.

Results. We detected a total of 151 radio sources. Among them, we identified a few radio sources whose flux density has apparently changed on timescales of months. Despite the limitations of our search, their possible variability makes these objects a top-priority target for multiwavelength studies of the potential counterparts of highly variable, unidentified gamma-ray sources.
\end{abstract}

Key words. radio continuum: stars - gamma rays: observations - X-rays: binaries

\section{Introduction}

The high-energy sky revealed by the present and past generations of $\gamma$-ray telescopes and satellites is populated by a large number of unidentified sources. For instance, the Third EGRET catalogue (Hartman et al. 1999) contains 271 entries and nearly two thirds of these $\gamma$-ray sources presently remain unidentified. At low galactic latitudes $\left(|b| \leq 10^{\circ}\right), 40$ of them do not show any positional coincidence (within the 95\% EGRET contour, i.e., a size of about $0.5-1^{\circ}$ ) with possible $\gamma$-ray objects known in our Galaxy (Romero et al. 1999; Torres et al. 2001a). Since it is most unlikely that all these sources are extragalactic, they should belong to one or more populations of galactic $\gamma$-ray sources yet

\footnotetext{
* Table 4 is only available in electronic form at the CDS via anonymous ftp to cdsarc.u-strasbg.fr (130.79.128.5) or via http://cdsweb.u-strasbg.fr/cgi-bin/qcat?]/A+A/482/247 $\star \star$ Member of CONICET.
}

to be discovered. In particular, both observational and theoretical arguments point to the idea of galactic sources of relativistic jets (e.g. microquasars and microblazars) being behind some of the EGRET unidentified sources. For instance, the high-mass X-ray binaries LS 5039 and LS I+61 303 have been reported as likely counterparts to 3EG J1824-1514 and 3EG J0241+6103, respectively (Kniffen et al. 1997; Paredes et al. 2000). Such an association has been strongly supported by the detection, at $\mathrm{TeV}$ energies, of LS 5039 by the High Energy Stereoscopic System (HESS, Aharonian et al. 2005) and of LS I+61 303 by the Major Atmospheric Gamma Imaging Cherenkov telescope (MAGIC, Albert et al. 2006). In addition, theoretical models have been developed that consistently explain the highenergy gamma-ray emission in terms of either external and synchrotron self-Compton processes in the jets (Kaufman-Bernadó et al. 2002; Bosch-Ramon et al. 2005) or hadronic interactions with wind material (Romero et al. 2003; Orellana et al. 2007). 
Table 1. Right ascension and declination (J2000.0) for the pointing centres of GMRT mosaics of the EGRET sources observed in this work.

\begin{tabular}{ccccc}
\hline \hline $\begin{array}{c}\text { Pointing } \\
\text { Id. }\end{array}$ & $\begin{array}{c}\text { 3EG J1735-1500 } \\
\left(\mathrm{hms},{ }^{\circ}{ }^{\prime \prime}{ }^{\prime \prime}\right)\end{array}$ & $\begin{array}{c}\text { 3EG J1746-1001 } \\
\left(\mathrm{hms},{ }^{\circ}{ }^{\prime \prime}{ }^{\prime \prime}\right)\end{array}$ & $\begin{array}{c}\text { 3EG J1810-1032 } \\
\left(\mathrm{hms},{ }^{\circ}{ }^{\prime \prime}\right)\end{array}$ & $\begin{array}{c}\text { 3EG J1904-1124 } \\
\left(\mathrm{hms},{ }^{\circ},{ }^{\prime \prime}\right)\end{array}$ \\
\hline 0 & $173552.80-150000.0$ & $174600.00-100148.0$ & $181004.80-103224.0$ & $190450.40-112512.0$ \\
1 & $173642.41-143912.6$ & $174648.69-094100.7$ & $181053.57-101136.7$ & $190539.31-110424.7$ \\
2 & $173732.19-145958.7$ & $174737.49-100147.1$ & $181142.45-103223.1$ & $190628.34-112511.0$ \\
3 & $173642.57-152046.7$ & $174648.80-102234.9$ & $181053.68-105310.8$ & $190539.43-114558.8$ \\
4 & $173503.03-152046.7$ & $174511.20-102234.9$ & $180915.92-105310.8$ & $190401.37-114558.8$ \\
5 & $173413.41-145958.7$ & $174422.51-100147.1$ & $180827.15-103223.1$ & $190312.46-112511.0$ \\
6 & $173503.19-143912.6$ & $174511.31-094100.7$ & $180916.03-101136.7$ & $190401.49-110424.7$ \\
\hline
\end{tabular}

Alternatively, both theroretical work (Dubus 2006) and observational data (Dhawan et al. 2006) have been used to claim that this emission may also come from a pulsar wind scenario in cases such as LS I+61 303. The jet or pulsar scenario remains at present a matter of interesting debate (Romero et al. 2007).

Here we will focus our attention on highly variable unidentified EGRET sources, defining the sample as those presenting a variability index $I \geq 2.5$ as given by Torres et al. (2001a), which by being a relative comparison places them more than $3 \sigma$ away from statistical variability of pulsars. Other variability indices have been introduced (see e.g., the $\delta$-index of Nolan et al. 2003). Although statistically correlated (Torres et al. 2001b), the specific classification of a given source can vary in each scheme, i.e., using the $I$ or $\delta$ indices. For the four sources herein analysed, the $\delta$-index is also compatible with they being gamma-ray variables. Variability is naturally expected in a microquasar/microblazar scenario due to several causes such as jet precession, motion in an eccentric orbit, and accretion rate changes due to stellar wind inhomogeneites, although such behaviour does not exclude that we are dealing with active galactic nuclei (AGNs), which are also known to be variable sources of $\gamma$-rays. Models also predict that these variations should be reflected not only in $\gamma$-rays but also in the jet non-thermal radio emission.

To determine the possible counterpart of selected EGRET sources, we undertook two campaigns with different radio interferometers to search for variable radio counterparts of a sample of variable unidentified EGRET sources. In the 2004 campaign we used the Westerbork Synthesis Radio Telescope (WSRT) for multiepoch radio observations of three of the most variable EGRET sources at low galactic latitudes, to determine that several radio variables were present in their location error box with their flux density changing in more than a $30 \%$ amplitude on timescales of months (Paredes et al. 2005). In the 2005 campaign, we conducted observations with the GMRT of the remaining four EGRET sources. This paper is devoted to presenting an account of these results.

\section{GMRT observations}

The observed fields, each about one square degree, were those corresponding to EGRET sources 3EG J1735-1500, 3EG J1746-1001, 3EG J1810-1032 and 3EG J1904-1124.

The radio observations were carried out with the GMRT of the National Centre for Radio Astrophysics (NCRA) in Khodad (India), during February 23 and April 19, 2005. The observations were made at the $1.4 \mathrm{GHz}$ frequency ( $21 \mathrm{~cm}$ wavelength) in spectral-line mode with 128 channels covering a $32 \mathrm{MHz}$ bandwidth, with two polarizations and two sidebands. The full-array synthesised beam of the GMRT interferometer was about 2-3" with the field-of-view limited by a $24^{\prime}$ full width half maximum
(FWHM) primary beam. To cover the larger $\left(\sim 1^{\circ}\right) \gamma$-ray error boxes with a sensitivity as uniform as possible, each EGRET source field was covered with a hexagonal pattern of 7 pointings. In this mosaicing approach, one pointing was centred on the nominal EGRET position and the other six offset by one primary beam FWHM with position-angle increments of $60^{\circ}$ in galactic coordinates. Their right ascension and declination are listed in Table 1. We devoted an integration time of about 20 min to each pointing. In this way, we were always able to map the whole solid angle of the EGRET $68 \%$ confidence contours and usually most of the $95 \%$, too. This technique was also applied when using the Very Large Array (VLA) and the WSRT to map the fields of 3EG J1928+1733, 3EG J2035+4441 and 3EG J1812-1316 (Paredes et al. 2005).

The calibration of amplitude and bandpass was achieved by observing 3C 286 and 3C 48, whereas phase calibration was performed through repeated scans of the nearby phase calibrators J1733-130, J1822-096, and J1911-201. The first two fields used both interleaved scans of J1733-130. Correction of the GMRT flux densities for the increase in the sky temperature in the direction of the pointings was also taken into account. The GMRT data was processed using standard procedures within the Astrophysical Image Processing System (AIPS) software package of NRAO. Self-calibration was possible in most of the pointings with some exceptions when no suitable bright sources were available in the field. The mosaicing of the pointings for each field was carried out using the FLATN task of AIPS, which includes weighting according to the primary beam response at each pixel position.

\section{Results}

We show in Fig. 1 an example of one of the obtained radio maps, which corresponds to a GMRT mosaic of the source 3EG J1735-1500. It was computed using uniform weight. We inspected each of the error boxes of the four EGRET sources observed and searched for all radio sources in the field. This was achieved through a combined use of visual inspection for those obvious cases and automated source extraction procedures, such as the Search and Destroy (SAD) task contained in the AIPS package. Only objects with peak flux densities higher than 4-5 times the root mean square (rms) noise were retained. For each radio source detected, we measured its peak $\left(S_{v}^{\text {Peak }}\right)$ and the total flux density $\left(S_{v}^{\text {Integ }}\right)$, position and angular size by means of an elliptical Gaussian fit. This procedure was carried out separately for the February 23 (first epoch) data, the April 19 (second epoch) data, and for the combined maps from both epochs. At the end of this process, all source detections were finally revised manually to ensure their reality. Artifacts near bright sources that could be considered low-significance detections by SAD were 


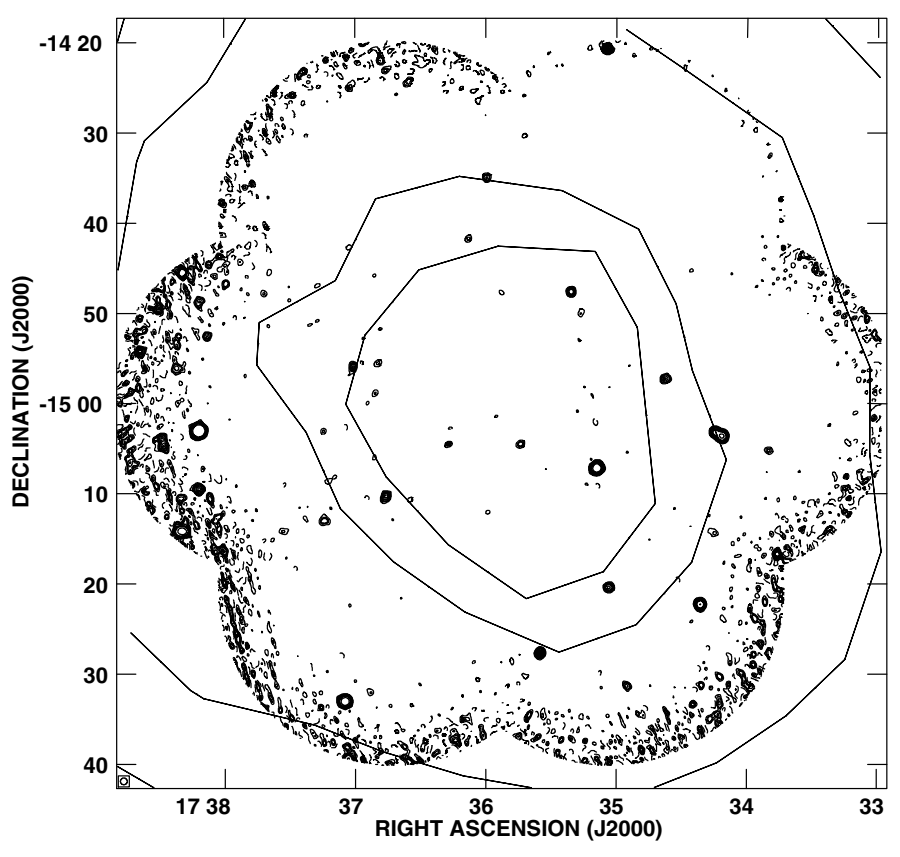

Fig. 1. The 1.4 GHz image of the error box of the unidentified EGRET source 3EG J1735-1500 obtained with the GMRT using a mosaicing technique. This image has been restored with a $45^{\prime \prime}$ circular beam for easier display purposes. Shown contours correspond to $-5,5,8,10,15$, $20,30,40,50$ and 100 times $0.4 \mathrm{mJy}_{\text {beam }}^{-1}$. The noisy appearance toward the edges is due to strong primary beam correction. The $50 \%$, $68 \%, 95 \%$, and $99 \%$ confidence contours for the location of the EGRET source are also shown. Forty-three radio sources are clearly detected in this field, but only two of them show any evidence of variability.

removed and the fitting of close double radio sources was also checked individually.

\subsection{Catalogue}

Based on the combined maps from the two observing epochs, we detected a total of 151 radio sources above a flux density of $\sim 1.5 \mathrm{mJy}$. Typical $\mathrm{rms}$ noises achieved in each pointing are in the 0.1-0.4 mJy range depending on particular observing conditions and bright sources limiting the dynamical range. The strongest source detected is GMRT J173811.6-150301, with an average peak flux density of $460.8 \mathrm{mJy}_{\text {beam }}{ }^{-1}$.

In Table 4, available in electronic form at the CDS, we list all the radio sources detected. From the 1st to 8th columns, this table contains the GMRT name for each entry, the J2000.0 coordinates, the peak flux density, the integrated flux density and the apparent angular size. The corresponding values were determined by fitting elliptical Gaussians using the AIPS task JMFIT. Uncertainties quoted in 4th and 5th columns are based on the formal errors of the fit and allow the reliability of the detection to be judged. However, they do not include the contribution of primary beam corrections as a function of angular distance $\theta$ to the phase centre (9th column). An estimate of the combined error is given in the 10th and 11th columns for both the peak and integrated flux density, respectively. The final 12 th column contains a radio variability index expressing the flux density difference between the two observing epochs in terms of this sort of combined noise estimator (see below and Appendix A for details.) This information has been omitted for a few cases where an unreliable result is suspected. This is usually connected with some extended or faint sources and occasional Gaussian fitting problems.
The GMRT positions are usually accurate to better than one arc-second, which is suitable for identifying optical/near infrared counterparts in follow-up observations even in relatively crowded fields. A preliminary search has been conducted in some cases by inspecting the plates from the Digitized Sky Survey (DSS, Lasker et al. 1990) and the 2 Micron All Sky Survey (2MASS, Paredes et al. 2005) as discussed below.

\subsection{Search for variable radio sources}

Based on previous radio work, such as the GT galactic plane patrol (Gregory \& Taylor 1986) or the FIRST survey (de Vries et al. 2004), one could expect that $1 \%$ to $5 \%$ of the sources we detected could be intrinsically variable. This would translate into one or two peculiar variable objects expected in this work.

The search for variables in the GMRT data was first carried out systematically by plotting the flux densities of sources detected at two different epochs (February and April), one versus the other. Of course, two epochs of observation are not sufficient to clearly establish an object's variability unless the amplitude of variation is rather high. However, this is the kind of object that we are looking for in this work as radio counterparts. In this context, a threshold of variability amplitude of $\pm 30 \%$, which is a substantial fraction of the total flux, appeared reasonable for our purposes.

One example of a variability plot is illustrated in Fig. 2 in the case of 3EG J1735-1500 based on our two epoch GMRT observation separated by a few months. Here, two radio sources in the field stand out as apparent radio variables. In the four EGRET fields observed, a total of 11 candidate variables were initially selected in this way. However, as quoted above, the absolute flux densities of our sources could be additionally affected by uncertainties in the primary beam correction applied to them. The net accuracy of pointing and tracking of GMRT antennas is about 2 to 3 arcmin, which at $L$-band corresponds to about $10 \%$ of the FWHM. This leads to poor primary beam correction resulting in significant uncertainties in the absolute flux densities of sources away from the pointing centre (see Appendix A). In view of this problem, we instead define a radio variability index measuring the significance of this difference between a source's individual peak flux densities from its average value $\bar{S}_{v}^{\text {Peak }}$ as

Var. Index $=\sqrt{\left[\frac{S_{v}^{\text {Peak }, 1}-\bar{S}_{v}^{\text {Peak }}}{\text { Err }^{\text {Peak }, 1}}\right]^{2}+\left[\frac{S_{v}^{\text {Peak }, 2}-\bar{S}_{v}^{\text {Peak }}}{\text { Err }^{\text {Peak }, 2}}\right]^{2}}$,

where the combined JMFIT + primary beam correction error is computed as

$\operatorname{Err}^{\text {Peak }, i}(\theta)=\sqrt{[\text { rms JMFIT }]^{2}+\left[S_{v}^{\text {Peak }, i} \frac{\Delta P_{b}}{P_{b}(\theta)^{2}}\right]^{2}}$

for each of the two observing epochs $(i=1,2)$. Here, the second term represents the error when dividing by the primary beam response $P_{b}(\theta)$ at the source location (Eq. (A.3)). If a source has a variability index above say 3 , we consider it as a candidate variable radio source.

Only two radio sources in our catalogue turned out to have a reliable variability index approaching 3 or higher, assuming a typical GMRT pointing error of $\Delta \theta=2^{\prime}$. The observed parameters of these candidate variables are separately listed in Table 2, bearing in mind that variability still needs to be confirmed. Notation here is similar to Table 4 . The variability indices for the rest of the GMRT radio sources are consistent 


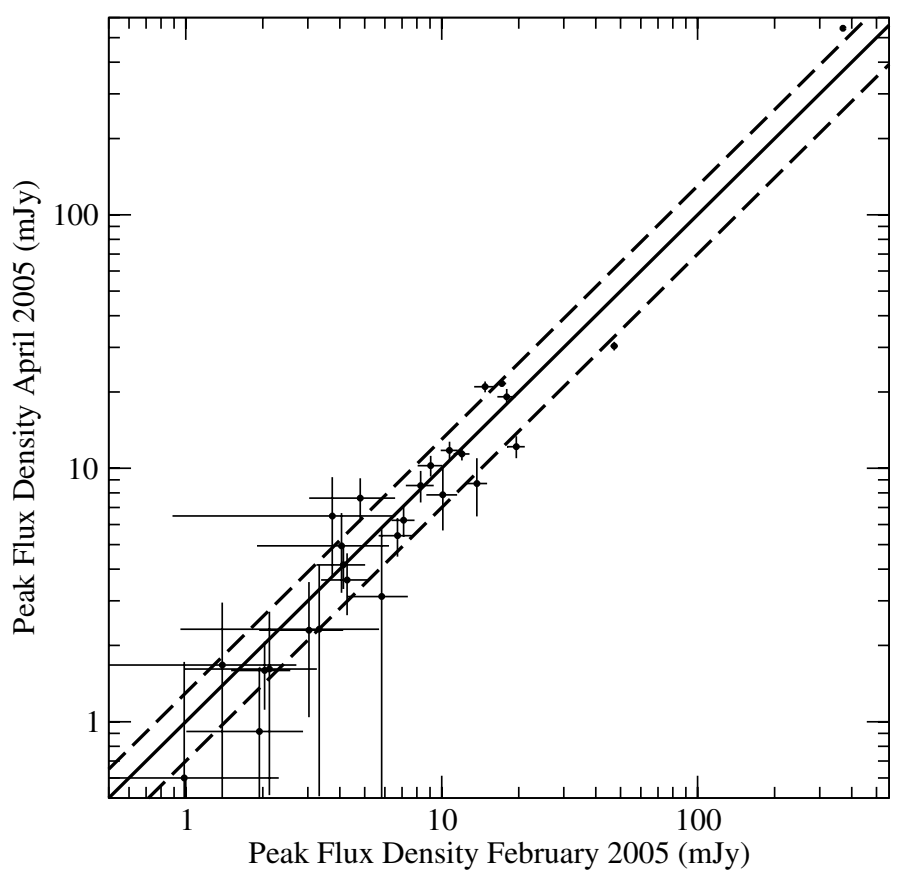

Fig. 2. Flux density in February 2005 versus flux density in April 2005 for all compact radio sources in the field of 3EG J1735-1500 detected with the GMRT at $21 \mathrm{~cm}$. Error bars shown are \pm 3 times the JMFIT rms. The dashed lines represent a variability amplitude of $\pm 30 \%$ above which an object is considered a likely radio counterpart of the unidentified variable EGRET source. Two radio sources in this field are found to satisfy such criterion but their large angular distance from the phase centre makes their variability suspicious due to uncertain primary beam correction.

with being non-variable. Of course, the possible detection of variability does not ensure an immediate connection with the corresponding EGRET source. Indeed, many extragalactic radio sources, such as quasars and other AGNs, are known to be variable in the radio. Despite the limitations of our variability analysis, the candidate variables reported here do represent good targets for follow-up observations that could ultimately reveal clues leading to the final identification of the EGRET source, especially if their position is confirmed with a better precision by future $\gamma$-ray telescopes such as the Gamma-ray Large Area Space Telescope (GLAST).

\subsection{Resolved and multiple radio sources}

We also looked for sources with a resolved structure. For each detection we compared the peak $S_{v}^{\text {Peak }}$ with the integrated flux density $S_{v}^{\text {Integ }}$ to differentiate among resolved and unresolved sources. The maps from the two epochs combined were used for this purpose. We plotted in Fig. 3 the ratio $S_{v}^{\text {Integ }} / S_{v}^{\text {Peak }}$ versus $S_{v}^{\text {Peak }}$. Following Bondi et al. (2007), we roughly estimated the lower envelope of the data shown in Fig. 3 by fitting the equation

$S_{v}^{\text {Integ }} / S_{v}^{\text {Peak }}=a^{-\left(b / S_{v}^{\text {Peak }}\right)}$

where $a=0.75$ and $b=-0.25$. This curve was later mirrored above with respect to the $S_{v}^{\text {Integ }} / S_{v}^{\text {Peak }}=1$ line. Data points in between the lower and upper mirrored envelopes are believed to correspond to point-like sources and to their dispersion due to statistical errors. In contrast, points lying above the upper envelope are considered as resolved sources. They represent about $23 \%$ out of the total detected sources, although this value must be taken as an upper limit because some of them are grouped as double sources. Double or triple morphologies are often found among them and none exhibited structural changes between the two epochs of observation.

\section{Discussion: individual EGRET fields}

\subsection{EG J1735-1500}

This is the most variable EGRET source in our sample $(I=8.86$, Torres et al. 2001a, see, however, Nolan et al. 2003). Inside its EGRET error box, Mattox et al. (2001) reported the presence of PMN J1738-1502, a source from the Parkes-MIT-NRAO survey (PMN, Griffith et al. 1994), as a potential radio counterpart with a low a priori probability that this object is a $\gamma$-ray blazar. In an analysis carried out later by Sowards-Emmerd et al. (2004), they classify this radio source as a high-confidence blazar. PMN J1738-1502 appears to be coincident with our variable source GMRT J173811.6-150301 in Table 2, with the observed variability in agreement with the proposed blazar nature. Combi et al. (2003) confirmed as well the presence of PMN J1738-1502 as a flat-spectrum compact radio source, with a flux density of $330 \mathrm{mJy}$ at $1.4 \mathrm{GHz}$. In addition, by using the NRAO VLA Sky Survey (NVSS; Condon et al. 1998) they found a total of 23 radio sources with flux density greater than $10 \mathrm{mJy}$ at $1.4 \mathrm{GHz}$ within the inner $95 \%$ confidence contour of 3EG J1735-1500.

In the Combi et al. (2003) exploration of the 3EG J1735-1500 error box, they also reported the presence a new radio galaxy (a double-sided source of Fanaroff-Riley type II) inside the $95 \%$ EGRET confidence contour. The radio galaxy's central core has an estimated position of $\alpha_{\mathrm{J} 2000.0}=17^{\mathrm{h}} 37^{\mathrm{m}} 12.9^{\mathrm{s}} \pm 0.3^{\mathrm{s}}, \delta_{\mathrm{J} 2000.0}=-15^{\circ} 11^{\prime} 0.20^{\prime \prime} \pm 15^{\prime \prime}$, but nothing is detected here in our GMRT maps. Actually, this is not totally unexpected since this position lies very close to mosaic-pointing edges where the primary beam correction increases the noise significantly. Based on the NVSS peak flux density, one would expect a source at merely 4-5 sigma level and; therefore, its non detection does not come as a surprise. On the other hand, removing the shortest GMRT baselines to enhance compact sources makes the radiogalaxy extended lobes fully resolved in our GMRT maps.

Our observations have revealed a total of 43 sources in this field, all listed in Table 4. Four of them have been resolved by the GMRT interferometer presenting either a double structure (GMRT J173345.7-151643, GMRT J173421.2-152222, and GMRT J173704.3-153301) or even a triple structure (GMRT J173412.8-150328). We show in Fig. 4 the contour map of this last interesting triple object, obtained after combining the February and April runs. The position of the components and their peak and integrated flux densities are given in Table 3. The morphology of this source thus appears clearly reminiscent of a Fanaroff-Riley type II radio galaxy, with a core and two lobe components. One of the lobes has a clear and bright hot spot. The three components are well-aligned in the northeast-southwest direction, with the outer components separated from the core by an angular distance of about $30^{\prime \prime}$.

No reliable radio variables have been detected in this field. Bosch-Ramon et al. (2006a) applied a microquasar model to explain the high-energy $\gamma$-ray emission of 3EG J1735-1500 consistent with the observations at lower energies (from radio frequencies to soft $\gamma$-rays) within the EGRET error box. Although their theoretical model suggests that a microquasar 
Table 2. Candidate variable radio sources detected with the GMRT inside the error box of one of our selected $\gamma$-ray variable EGRET sources.

\begin{tabular}{|c|c|c|c|c|c|c|c|c|c|}
\hline $\begin{array}{l}\text { Source } \\
\text { 3EG J }\end{array}$ & GMRT Id. & $\begin{array}{c}\alpha_{\mathrm{J} 2000.0} \\
(\mathrm{hms})\end{array}$ & $\begin{array}{c}\delta_{\mathrm{J} 2000.0} \\
\left({ }^{\circ},{ }^{\prime \prime}\right)\end{array}$ & $\begin{array}{c}\theta \\
\left({ }^{\prime}\right)\end{array}$ & $\begin{array}{r}S_{v}^{\text {Peak }, 1} \\
(\mathrm{mJy} / \mathrm{b})\end{array}$ & $\begin{array}{l}\text { ErrPeak,1 } \\
(\mathrm{mJy} / \mathrm{b})\end{array}$ & $\begin{array}{r}S_{v}^{\text {Peak, } 2} \\
(\mathrm{mJy} / \mathrm{b})\end{array}$ & $\begin{array}{c}\text { ErrPeak,2 } \\
\mathrm{mJy} / \mathrm{b}\end{array}$ & $\begin{array}{l}\text { Var. } \\
\text { Index }\end{array}$ \\
\hline \multirow[t]{2}{*}{$1904-1124$} & J190601.7-112510 & $90601.781(0.009)$ & $-112510.10(0.20)$ & 6.5 & $5.1 \pm 0.3$ & 0.8 & $9.1 \pm 0.6$ & 1.5 & 3 \\
\hline & J190617.4-112850 & 1906 17.428(0.003) & $-112850.11(0.04)$ & 4.5 & $16.2 \pm 0.3$ & 1.5 & $26.1 \pm 0.5$ & 2.4 & 4 \\
\hline
\end{tabular}

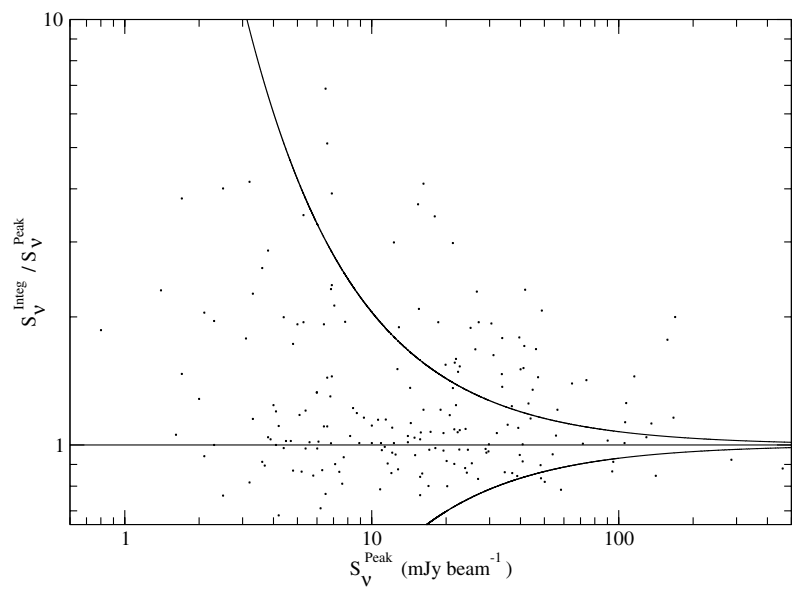

Fig. 3. Plot of the integrated to peak flux density ratio for all detections in this work as a function of peak flux density. Continuous lines represent the upper and lower envelopes of the plot region considered to correspond to unresolved radio sources according to an approach similar to the one in Bondi et al. (2007). Objects located above the upper envelope line appear to be resolved by our GMRT observations.

might be the counterpart of this particular source, other alternatives cannot be ruled out as possible counterparts (e.g., Punsly et al. 2000; Bosch-Ramon et al. 2006b).

\subsection{EG J1746-1001}

We have detected 36 sources within the error box of this EGRET source. Two of them were reported by Mattox et al. (2001) as potential radio counterparts of 3EG J1746-1001 with a low a priori probability. These sources are PMN J1744-1011 and PMN J1747-0959 with a flux density at $5 \mathrm{GHz}$ of 85 and $61 \mathrm{mJy}$, respectively. We have also detected these sources at $1.4 \mathrm{GHz}$ showing a double structure.

For the first one PMN J1744-1011, we have resolved an elongated northeast-southwest structure with two strong components. GMRT J174443.2-101001 is the northern component, with a peak flux density of $46.2 \pm 0.7 \mathrm{mJy}^{-1}$ beam $^{-1}$ and an integrated flux density of 77.6 \pm 1.4 mJy. GMRT J174441.9-101040 is the southern component, which appears with a peak flux density of $106.3 \pm 0.7 \mathrm{mJy}^{-1}$ beam $^{-1}$ and an integrated flux density of $120.3 \pm 1.1 \mathrm{mJy}$. For the second one PMN J1747-0959, our GMRT map shows a double source elongated in the east-west direction. The east component, GMRT J174727.8-095917, has a peak flux density of $129.4 \pm 0.4 \mathrm{mJy} \mathrm{beam}^{-1}$ and an integrated flux density of $134.8 \pm 0.6 \mathrm{mJy}$. The peak and the integrated flux density of the western component, GMRT J174727.2-095911, amount to $12.1 \pm 0.4 \mathrm{mJy}_{\text {beam }}{ }^{-1}$ and $13.3 \pm 0.6 \mathrm{mJy}$, respectively.

We also found other five sources in this field showing extended or double structures, namely GMRT J174457.8-101206, GMRT J174501.4-093849, GMRT J174556.0-100613, GMRT J174616.5-102358, and GMRT J174624.1-095208. Information about their peak and integrated flux densities,

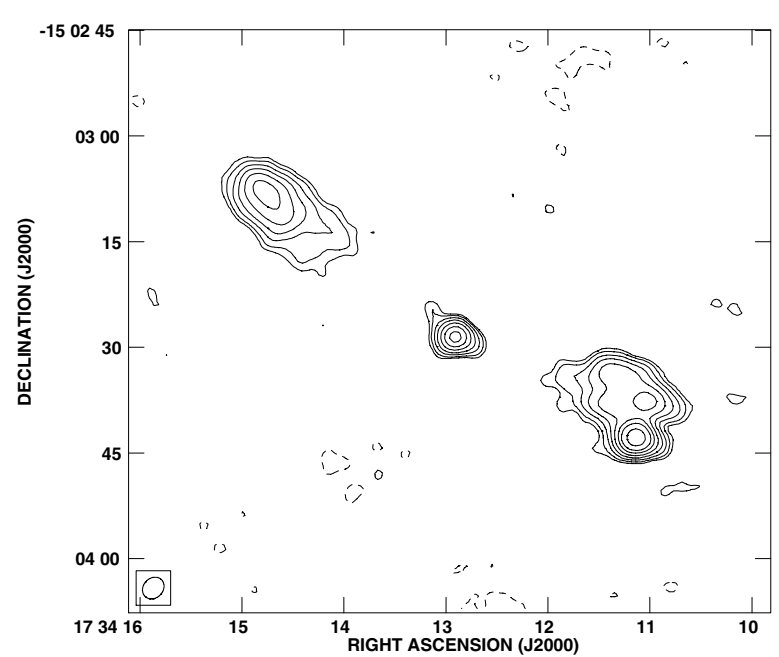

Fig. 4. Triple radio source in the field of the unidentified EGRET source 3EG J1735-1500 obtained after combining the February and April runs with the GMRT. The contours shown are $-3,3,5,9,15,25,40,60,100$ and 150 times the rms noise of $0.13 \mathrm{mJy}$ beam $^{-1}$. The corresponding synthesised beam is shown in the bottom left corner, and it corresponds to 3 ." $46 \times 2$ ". 91 , with a position angle of -36.8 .

apparent size, and position angle of their components can be found in Table 4.

No reliable variables were found in the field of 3EG J1746-1001. An interesting object is, however, GMRT J174535.5-101439, which has a barely resolved core+one-sided jet morphology. No obvious counterpart is present at optical, infrared, or X-ray according to the inspected surveys.

\subsection{EG J1810-1032}

We detected 38 sources within the error box of this EGRET source. For this $\gamma$-ray source, three different potential radio counterparts with low a priori probability were proposed by Mattox et al. (2001). These sources were PMN J1808-1041, PMN J1810-1054, and PMN J1810-1102 with a flux density at $5 \mathrm{GHz}$ from single-dish surveys of 48, 49, and $103 \mathrm{mJy}$, respectively. We have not clearly detected any of them and this is likely due to lack of sensitivity to very extended objects more easily detected in single dish surveys.

Among the 38 sources detected, there are five of them that have been resolved with the GMRT showing a double or marginally resolved double structure. These sources are GMRT J180809.1-104031, GMRT J180834.3-103024, GMRT J180943.4-104055, GMRT J181017.7-102907, and GMRT J181030.9-101839.

No reliable radio variables were found in this field. 
Table 3. The triple radio source within the error box of 3EG J1735-1500.

\begin{tabular}{ccccc}
\hline \hline Component & $\begin{array}{c}\alpha_{\mathrm{J} 2000.0} \\
(\mathrm{~h} \mathrm{~m} \mathrm{~s})\end{array}$ & $\begin{array}{c}\delta_{\mathrm{J} 2000.0} \\
\left({ }^{\circ}{ }^{\prime \prime}\right)\end{array}$ & $\begin{array}{c}S_{v}^{\text {Peak }} \\
\left(\mathrm{mJy} \mathrm{beam}^{-1}\right)\end{array}$ & $\begin{array}{c}S_{v}^{\text {Integ }} \\
(\mathrm{mJy})\end{array}$ \\
\hline West lobe $^{(*)}$ & $173411.126(0.002)$ & $-150342.65(0.03)$ & $21.9 \pm 0.4$ & $27.1 \pm 0.7$ \\
Core & $173412.899(0.003)$ & $-150328.55(0.05)$ & $12.5 \pm 0.4$ & $11.8 \pm 0.6$ \\
East lobe & $173414.720(0.020)$ & $-150309.00(0.20)$ & $6.6 \pm 0.4$ & $34.0 \pm 2.0$ \\
\hline
\end{tabular}

(*) This lobe is decomposed into two source components in Table 4.

\subsection{EG J1904-1124}

We detected 34 sources within the error box of this EGRET source. Two different potential radio counterparts, with a low a priori probability, were proposed by Mattox et al. (2001). These sources were PMN J1905-1153 and PMN J1906-1114 with a flux density at $5 \mathrm{GHz}$ of 197 and $126 \mathrm{mJy}$, respectively.

The first of them has been classified as a plausible blazar by Sowards-Emmerd et al. (2004). We also detected this source, with a peak flux density of $286.2 \pm 1.4 \mathrm{mJybeam}^{-1}$ and an integrated flux density of $264.2 \pm 2.0 \mathrm{mJy}$. These peak and integrated flux densities fall slightly offset from the envelope curve quoted in Sect. 3.3, but yet they can be considered to be consistent with an unresolved source. Although our data at $1.4 \mathrm{GHz}$ and the $5 \mathrm{GHz}$ data were not taken simultaneously, the results seem to point to a non-thermal emission. The second source, PMN J1906-1114, also detected by us, shows an elongated structure with two components. The strongest one is GMRT J190645.1-111434, with a peak flux density of $115.7 \pm$ $0.7 \mathrm{mJy} \mathrm{beam}^{-1}$ and an integrated flux density of $167.6 \pm 1.3 \mathrm{mJy}$. The fainter component is GMRT J190644.8-111416 with a peak and an integrated flux density of $33.6 \pm 0.7 \mathrm{mJy} \mathrm{beam}^{-1}$ and $49.3 \pm 1.3 \mathrm{mJy}$, respectively. In this source, the northern component could have a non-thermal nature, whereas it is not as clear for the southern component. In the error box of 3EG J1904-1124, we also detected four other extended sources, namely GMRT J190339.9-114756, GMRT J190341.1-112310, GMRT J190430.2-115241 and GMRT J190523.0-110250.

In the field of 3EG J1904-1124, we found two sources that are candidate radio variables. Their expected flux density uncertainty due to primary beam correction is at the $\sim 10 \%$ level. Both of them have a compact appearance.

\section{Conclusions}

We have reported the radio results of source detection and of the search for variables positionally consistent with the highly variable, unidentified EGRET sources, at the $21 \mathrm{~cm}$ wavelength, and using a mosaicing technique with GMRT. The targets studied in this work include 3EG J1735-1500, 3EG J1746-1001, 3EG J1810-1032 and 3EG J1904-1124. Our main findings can be summarised as follows,

1. The number of confident detections within the error box of each EGRET source quoted above was $43,36,38$, and 34 objects, respectively. Our limiting flux density is not uniform across all pointings but typically amounts to $\sim 1.5 \mathrm{mJy}$. The mapped field of view always covered the full solid angle of the $68 \%$ EGRET confidence contours and usually most of the $95 \%$ as well.

2. Out of the 151 sources detected in total, only a few of them displayed apparent radio variability with reliable amplitude on a two-month time baseline in two of the EGRET fields.
Although a variability search was the original motivation for this work, we have been severely limited by instrumental problems in this task, and not all the detected sources could be suitably explored in their time behaviour. Nevertheless, we have undertaken a programme for future follow-up observation of the proposed candidate variables, aimed in particular at finding their near-infrared and X-ray counterparts. Although we anticipate here that most will turn out to be unrelated background sources, strong attention should be given to any of them found to be positionally coincident with the $\gamma$-ray emission detected by future space observatories such as GLAST with improved source localization

3. As a byproduct of this work, we have identified 16 double radio sources, 4 marginally resolved double sources, and one interesting triple radio source positionally consistent with 3EG J1735-1500. Its morphology is remarkably reminiscent of a Fanaroff-Riley type II radio galaxy. However, the absence of an optical/near infrared counterpart for the central core at present precludes assessing its true nature and connection with the EGRET emission.

Acknowledgements. The authors acknowledge support by the Ministerio de Educación y Ciencia (Spain) under grants AYA2007-68034-C03-01, AYA2007-68034-C03-02, AYA2006-00530, FEDER funds and Plan Andaluz de Investigación, Desarrollo e Innovación (PAIDI) of Junta de Andalucía as research group FQM322. D.F.T. also acknowledges partial support from the Guggenheim Foundation. G.E.R is supported by the Argentine Agencies CONICET (PIP 5375) and ANPCyT (PICT 03-13291). J.A.C. is a researcher of the programme Ramón y Cajal funded jointly by the Spanish Ministerio de Educación y Ciencia (former Ministerio de Ciencia y Tecnología) and Universidad de Jaén. During this work, V.B-R. has been supported by the DGI of the Ministerio de Ciencia y Tecnología (Spain) under the fellowship FP-20012699. V.B-R. thanks the Max-Planck-Institut für Kernphysik for its support and kind hospitality. V.B-R. gratefully acknowledges support from the Alexander von Humboldt Foundation. GMRT is run by the National Centre for Radio Astrophysics of the Tata Institute of Fundamental Research. We thank an anonymous referee for useful comments that helped to improve this paper.

\section{Appendix A: Uncertainty in absolute flux density due to pointing/tracking offsets}

The properties of the GMRT antennae are such that pointing/tracking offsets of a few arcmin are not uncommon. Consequently, the true primary beam pattern can experience a shift in this order instead of being centred exactly at the assumed phase centre. This effect is negligible at most of the typical GMRT long wavelengths, but not completely at the shortest one of $21 \mathrm{~cm}$ used here where it can reach about $10 \%$ of the primary beam FWHM.

How does this translate into primary beam correction? The anntena primary beam response $P_{b}(\theta)$ as a function of the distance $\theta$ from the phase centre can be computed using polynomic coefficients from the GMRT User's Manual. In general, it can be expressed as

$P_{b}(\theta)=1+A x^{2}+B x^{4}+C x^{6}+D x^{8}$ 


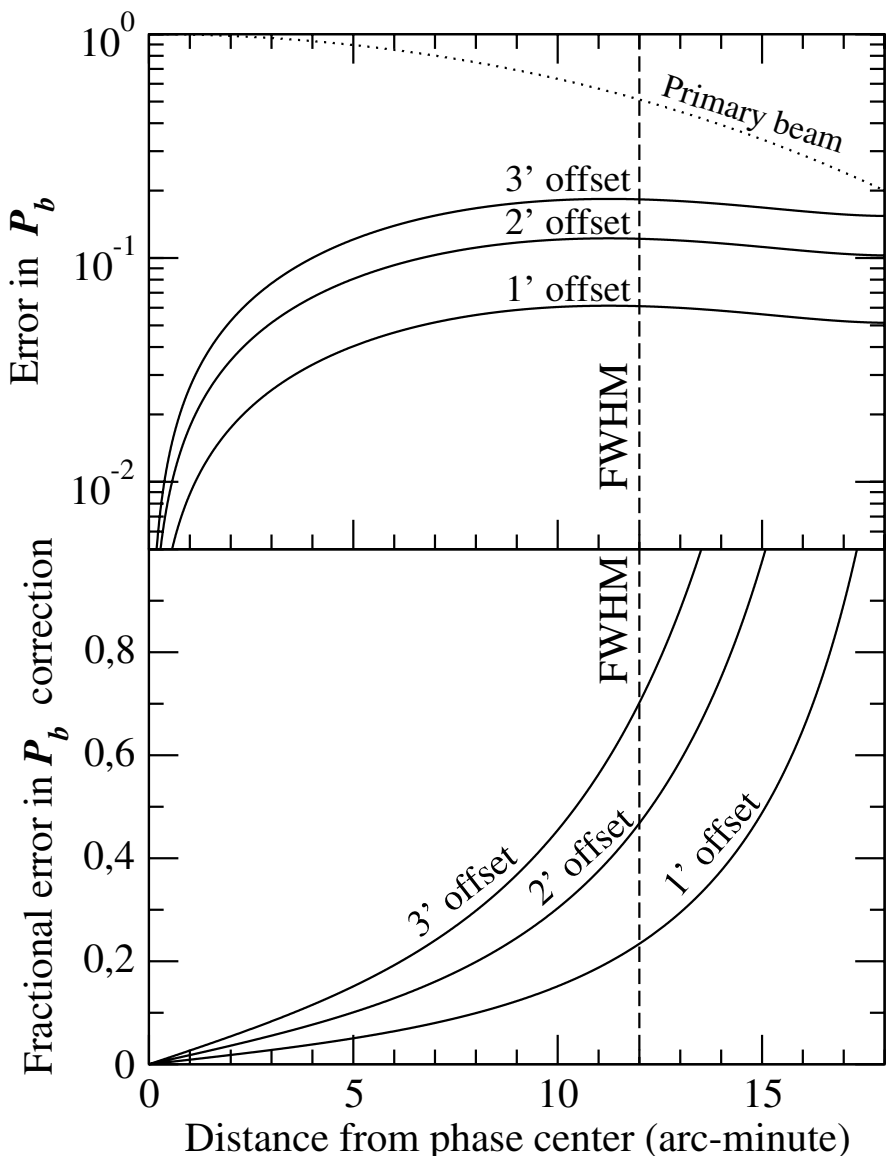

Fig. A.1. Top: uncertainty in the GMRT primary beam response at the $21 \mathrm{~cm}$ wavelength for different values of the pointing/tracking offset as a function of the distance from the phase centre. The shape of the primary beam is also plotted as a pointed line and the vertical dashed line indicates its FWHM. Bottom: fractional error introduced when the synthesised map is divided by the sky position dependent primary beam response shown in the top panel.

where $A=-2.27961 \times 10^{-3}, B=21.4611 \times 10^{-7}, C=$ $-9.7929 \times 10^{-10}, D=1.80153 \times 10^{-13}$, and $x=v \theta$ is the product of the observing frequency in $\mathrm{GHz}$ times the angular distance in arcmin. Any pixel in a synthesised radio map will have its distance to the phase centre known within an uncertainty $\Delta \theta$. We assume here that this value is comparable to the typical GMRT pointing/tracking offset. Therefore, the corresponding uncertainty in the primary beam response will propagate by incrementing Eq. (A.1) as

$\Delta P_{b}(\theta)=2 x\left(A+2 B x^{2}+3 C x^{4}+4 D x^{6}\right) \Delta x$, where $\Delta x=v \Delta \theta$, since we assume that the frequency is known exactly. Hereafter, we adopt $v=1.4 \mathrm{GHz}$ as the corresponding value for the $L$-band wavelength of $21 \mathrm{~cm}$.

To quantify the problem, the top panel in Fig. A.1 shows the error in primary beam response $\Delta P_{b}(\theta)$ computed using Eq. (A.2) as a function of $\theta$ for different offset values. The bottom panel illustrates the corresponding fractional error,

$\frac{\Delta S_{v}}{S_{v}}=\frac{\Delta P_{b}}{P_{b}^{2}}$,

when dividing a measured flux density $S_{v}$, or a map pixel value, by the primary beam response. From these plots we see that primary beam corrected flux densities may be affected by an additional uncertainty as large as $\pm 10 \%$ for objects beyond $\sim 6^{\prime}$ from the phase centre.

\section{References}

Aharonian, F. A., et al. 2005, Science, 309, 746

Albert, J., Aliu, E., Anderhub, H., et al. 2006, Science, 312, 1771

Bondi, M., et al. 2007, A\&A, 463, 519

Bosch-Ramon, V., Romero, G. E., \& Paredes, J. M. 2005, A\&A, 429, 267

Bosch-Ramon, V., Paredes, J. M., Romero, G. E., \& Torres, D. F. 2006a, A\&A, 446, 1081

Bosch-Ramon, V., Romero, G. E., \& Paredes, J. M. 2006b, A\&A, 447, 263

Combi, J. A., Romero, G. E., Paredes, J. M., Torres, D. F., \& Ribó, M. 2003, ApJ, 588, 731

Condon, J. J., et al. 1998, AJ, 115, 1693

de Vries, W. H., Becker, R. H., White, R. L., \& Helfand, D. J. 2004, AJ, 127, 2565

Dhawan, V., Mioduszewski, A., \& Rupen, M. 2006, PoS, Proceedings of the VI Microquasar Workshop, 52.1

Dubus, G. 2006, A\&A, 2006, 456, 801

Gregory, P. C., \& Taylor, A. R. 1986, AJ, 92, 371

Griffith, M. R., Wright, A. E., Burke, B. F., \& Ekers, R. D. 1994, ApJSS, 90, 179 Kaufman-Bernadó, M. M., Romero, G. E., \& Mirabel, I. F. 2002 A\&A, 385, L10

Kniffen, D. A., Alberts, W. C. K., Bertsch, D. L., et al. 1997, ApJ, 486, 126

Hartman, R. C., Bertsch, D. L., Bloom, S. D., et al. 1999, ApJS, 123, 79

Lasker, B. M., et al. 1990, AJ, 99, 2019

Mattox, J. R., Hartman, R. C., \& Reimer, O. 2001, ApJS, 135, 155

Nolan, P., Tompkins, W., Grenier, I., \& Michelson, P. 2003, ApJ, 597, 615

Orellana, M., Bordas, P., Bosch-Ramon, V., Romero, G. E., \& Paredes, J. M. 2007, A\&A, 476, 9

Paredes, J. M., Martí, J., Ribó, M., \& Massi, M. 2000, Science, 288, 2340

Paredes, J. M., Martí, J., Torres, D. F., et al. 2005, Ap\&SS, 297, 223

Punsly, B., Romero, G. E., Torres, D. F., \& Combi, J. A. 2000, A\&A, 364, 552 Romero, G. E., et al. 1999, A\&A, 348, 868

Romero, G. E., Torres, D. F., Kaufman-Bernadó, M. M., \& Mirabel, I. F. 2003, A\&A, 410, L1

Romero, G. E., Okazaki, A. T., Orellana, M., \& Owocki, S. P. 2007, A\&A, 474, 15

Sowards-Emmerd, D., Romani, R. W., Michelson, P. F., \& Ulvestad, J. S. 2004, ApJ, 609, 564

Skrutskie, M. F., et al. 2006, AJ, 131, 1163

Torres, D. F., Romero, G. E., Combi, J. A., et al. 2001a, A\&A, 370, 468

Torres, D. F., Pessah, M. E., \& Romero, G. E. 2001b, Astron. Nachr., 322, 223 